\title{
A NEW TESTING MACHINE FOR THE DYNAMIC CHARACTERIZATION OF HIGH STRENGTH LOW DAMPING FIBER MATERIALS
}

Rosario Ceravolo ${ }^{1}$, Andrea De Marchi $^{2}$, Elena Pinotti ${ }^{1}$, Cecilia Surace $^{1}$, Luca Zanotti Fragonara $^{3}$

${ }^{1}$ Politecnico di Torino, Department of Structural, Building and Geotechnical Engineering

Corso Duca degli Abruzzi 24, Turin 10129, Italy

${ }^{2}$ Politecnico di Torino, Department of Electronics and Telecommunications

Corso Duca degli Abruzzi 24, Turin 10129, Italy

${ }^{3}$ Cranfield University, School of Aerospace, Transportation and Manufacturing, Centre for Autonomous and Cyberphysical Systems

College Road, Cranfield MK43 0AL, United Kingdom

Corresponding author e-mail: cecilia.surace@polito.it, Telephone number +390110904904, Fax number: $+390110904899$

This work was partly supported by the US Department of Commerce within the NIST Precision Measurement Grant Program (Award ID \# 70NANB15H348)

Keywords Dynamic characterization, Prototyping testing machine, Non-linearity, Damping, Para-aramid, Silicon Carbide

\begin{abstract}
This work describes a novel method for measuring the damping, the elastic modulus and the non-linear behavior of high strength low damping fiber materials such as para-aramids, silicon carbide (SiC) and carbon. The method is based on resonant response characterization of a spring-mass system excited by a sine-wave forcing term which is applied as a vertical force to the suspended mass. The damping is obtained from the measured resonance quality factor $Q$, the elasticity modulus is calculated from the resonance frequency, and the non-linear coefficient is obtained with the backbone approach from resonance profile variations as a function of the forcing term amplitude. It is argued that the method is very sensitive, to the point that a maximum excitation amplitude of the order of a few percent of resistance is sufficient to obtain an estimate of the non-linear coefficient. This claim is supported by experimental results. A testing machine is also discussed, which provides the necessary sensitivity at such small excitation amplitudes and the capability of evaluating very small damping values, as expected in high strength low damping fiber materials. The sensitivity is guaranteed by an optical position sensor with sub-micron resolution. To evaluate small damping values,
\end{abstract}


particular care has been taken to ensure that energy dispersions in the generator are much smaller than energy dispersions in the fibers themselves. Examples of dynamic characterization are shown for para-aramid, silicon carbide, and carbon fibers.

\section{Introduction}

Fiber materials have recently attracted growing interest as they can be used in many different ways and in different fields. The most widespread applications regard signal transmission (e.g. optical fibers [1, 2]), force transmission (e.g. fibers in composites materials), protection and coating (e.g. textile tissue). In all cases, whether they are embedded in a matrix or not, they are subject in use to deformation and vibrations. It is then very important to study the dynamic behavior of the fibers and in particular their damping characteristics because they influence their dynamic response and consequently their efficiency.

This work was prompted by unexpectedly converging interests of two very distant worlds of Structural Engineering and Metrology of small forces, in particular for Gravitational Metrology applications. In both disciplines, high strength fibers have been identified as critical components with high potential for significant advancements of the field, and the lack of data on some of their relevant features is felt as a hindrance toward their full exploitation. A fruitful collaboration hence developed from initial discussions between the Structural Engineering and the Metrology groups of Politecnico di Torino, which eventually generated a common experimental effort for the measurement of fiber qualities that are not found in literature, but are relevant in the two fields.

In particular, structural engineers were interested in information about mechanical damping in high strength fibers in order to evaluate their energy absorption capability, clarify the effects of weaving on their behavior, and inquire into the possibility of using non-destructive tests for their characteristics (including damping and non-linearity) for damage assessment purposes. Nevertheless multifilament tows have attracted the least attention in the literature, which makes it hard to perform any comparisons. Metrology scientists, on the contrary, are interested in ideal structures to minimize Type B uncertainties [3], and need to assess the validity limits of their models. They like high strength fibers because they appear suitable by and large to fit the bill, and are interested in stability, $Q$ factor, and non-linearity. The ideal experiment would be designed for single fiber evaluation, a compromise of multifilament tows of parallel fibers would reduce systematic errors as long as the relevance of single fiber data is not lost.

Several techniques are described in literature for testing the damping behavior of composite materials. Research on freestanding fibers is less common. As a result, data for dynamic characteristics (e.g. damping) of free-standing fibers are 
not easily available in the open literature, while they might be very useful in assessing ageing or damage, or the dynamic response of composite materials.

In fact, standard equipment used to determine mechanical and dynamic properties is frequently unsuitable for the investigation of thin fibres and yarns. A universal testing machine for accurate dynamic characterization of thin fibers with diameter in the order of $10 \mu \mathrm{m}$ or a yarn does not exist because of restrictions on sample geometry, and difficult handling and clamping. This research suggests a possible solution to these problems.

Existing experimental methods potentially applicable to determine the dynamic moduli and damping of composite materials include the free vibration (impulse technique), the rotating-beam deflection, the forced vibration response, the resonance technique, and the continuous wave or pulse propagation technique $[4,5,6]$. One of the most popular tests is the impulse technique, which has been used, for example, to measure natural frequencies and loss factors [7]. The input is given by an instrumented impact hammer and the response is captured by an accelerometer and is typically read by an acquisition card [8]. In some cases, the impulse response is visualized by means of a high speed camera [9, 10]. The impulse method was initially proposed for the characterization of composites by Suarez et al. [11], Suarez and Gibson [12] and Crane and Gillespie [13], but these studies have not been applied to fibers. A hurdle which clearly arises due to geometrical reasons towards the application of the impulse technique to high stiffness fibers is the requirement for high sensitivity of the vibration amplitude sensor. Di Carlo and Williams [14] tested the damping properties of boron and silicon carbide fibers with a free-decay technique. They adopted a capacitive sensor in which the fiber was part of the capacitor and, in spite of its limited dimensions, they obtained usable results. However, this method cannot be applied to non-conductive materials.

Resonance techniques, as used in Dynamic Mechanical Analysis (DMA), consist of exciting a mechanical system at its eigen-frequency and measuring the motion in order to extract the damping parameters. Often DMA is used to evaluate the viscoelastic behavior of composite materials [15], as well as temperature changes [7]. Gibson et al. [11, 16, 17] measured the longitudinal damping of a fiber held in tension by fixing its upper end, hanging it down vertically, and securing a mass on its lower end. The measurement requires a mass to be fixed at one extremity of the fiber, thus leading to longitudinal pre-stretch of the fiber, which, in turn, influences the outcome of the measurement.

The work described in this paper fits into the category of resonant techniques. The aim of this work is the implementation of a method for the dynamic characterization of free-standing, high strength, and low damping fiber materials, such as para-aramids, silicon carbide ( $\mathrm{SiC})$ and carbon, including the detection of a possible non-linear behavior. The dynamic characterization consists of driving the fiber sample with a low intensity dynamic excitation 
force, and recording the response in terms of displacement as a function of frequency and amplitude. The results allow for derivation of the elastic modulus from the resonant frequency, the damping from the quality factor $Q$, and the nonlinearity factor from the backbone analysis of resonance response distortions as a function of the excitation amplitude.

The main problem in measuring very small damping values in high strength low damping fiber materials is the necessity to use equipment in which energy dispersions are very low, much smaller than dispersions in the fiber, including the sample holder, the measurement system and the exciter.

To solve this problem, a dedicated testing machine was developed at the Metrology Laboratory of the Department of Electronics and Telecommunications of Politecnico di Torino. The machine consists of a spring-mass resonator, where the spring is the fiber or fiber bundle under test, and the suspended mass is designed to conveniently preload the fibers. A web of thin (fiber) "tie-rods" constrains the mass to the single vertical degree of freedom, in order to keep low dissipation. A high sensitivity non-contact optical position detector was adopted for the same low dissipation reason, to measure the vertical displacements. Measurements are taken at resonance, as the system is excited by a low dissipation home-made non-contact voice-coil transducer.

In this work, the principle of operation of the testing apparatus and the design parameters are first discussed, then the prototype itself is described and finally, in the last section, experimental results on the dynamic characterization of paraaramid, silicon carbide $(\mathrm{SiC})$ and carbon fibers are reported and discussed.

\section{The resonant spring-mass approach}

When testing mechanical properties of fibers via a spring-mass approach, all individual fibers in a sample are subjected to the same tension at all times during both static and dynamic tests. The preload imposed by a hanging mass can be used to select the operating point on the stress-strain curve. Moreover, it guarantees that the fibers in the bundle never unload during an oscillation cycle. Consequently, if the non-linearities are small, the damping results obtained from a single unwoven bundle are expected to be representative of the single fiber behavior. Because no deconvolution is necessary and the experimental conditions are well defined, highly accurate information of the relevant material properties may be obtained directly from such measurements.

For example, by introducing appropriate constraints to the system, it is possible to reduce disturbances to the system and evaluate the resonant frequency with high accuracy. This parameter can then be used to determine the stiffness of the suspension and hence the elastic modulus $E$ of the material, as long as both the number and the diameter of the 
fibers are known. In the present work, the movement of the mass was restricted to a single vertical degree of freedom by means of a web of fibers, minimizing their contributions to both resonance frequency and losses.

The operating principles of the harness web in the prototype machine are described in some detail because they are a fundamental aspect of the proposed approach. The basic idea is to exploit the cosine law to minimize the stretching imposed on the constraining fibers (hereafter called "tie-rods") when a mass oscillates in the vertical direction. Accordingly, the tie-rods are attached to the mass horizontally, as shown in Fig. 1, in order for their stiffness to inhibit lateral displacements but allow for vertical ones.

The effective additional stiffness $k_{t z}$ introduced by the $n$ tie-rods to vertical oscillations is reduced in such a scheme, with respect to the total tie-rods stiffness $n k_{t}$, by the strain $\varepsilon_{t 0}$ produced in the tie-rods due to pre-tension when they are horizontal, plus half the square of their inclination angle $\theta$, as expressed by the following equation

$k_{t z} \approx n k_{t}\left(\frac{1}{2} \theta^{2}+\varepsilon_{t 0}\right)$

which can be easily derived from the analysis of the model shown in Fig. 1.

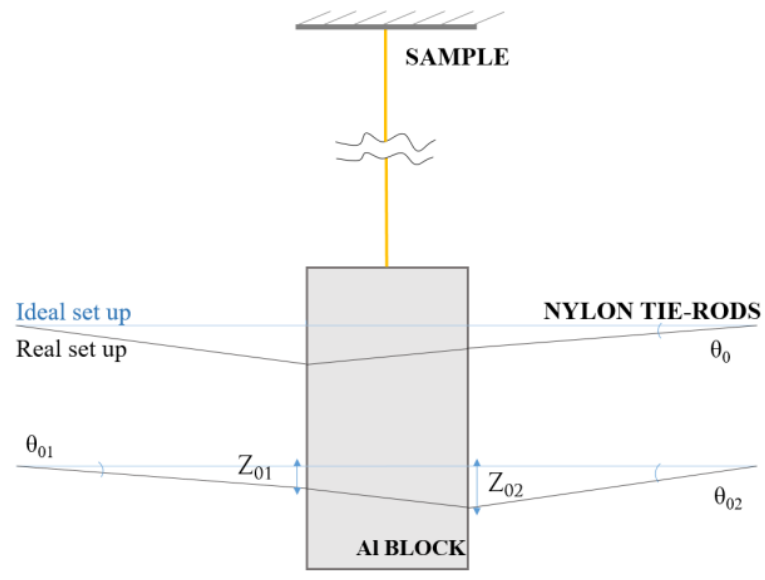

Fig. 1 Schematic arrangement of tie-rods, with attachment errors

In turn, the lateral stiffness to horizontal movements of the mass is given by $n k_{t} / 2$ for each orthogonal direction, which suggests that careful alignment, a suitable choice of material and imposed tension are key for optimization of the tie-rod web's performance. The lateral stiffness should be high, so that the pendulum mode frequency is much higher than the vertical spring-mass mode frequency of interest, but the vertical stiffness contribution from the harness should be low in order to minimize the effect on resonance frequency and damping. As discussed below, it is much easier to guarantee this condition for a multifilament bundle than for a single fiber, and this is the reason for the choice made to avoid single fiber measurements in this initial phase of development. 
The practical implementation of tie-rods in the prototype machine is shown in Fig. 2, where the mechanism adopted to fix the tie-rods' tension is also visible. This mechanism consists of sixteen $0.13 \mathrm{~kg}$ bolts suspended at the extremity of a tie-rod beyond a pulley fixed on a post analogously to the Atwood machine. If all pulleys are carefully positioned at the same level above the table, and the tie-rods are glued to the mass only after all parts settle down, only the residual long term creep in the fibers under testing can produce sizeable values of the angle $\theta$ in equation (1), which can be otherwise expected to remain below a couple of milliradians and, therefore, contribute negligibly to the stiffness in comparison to the effect of strain. Similarly, small dynamic variations in $\theta$ during oscillation are expected to have a negligible effect on the stiffness and hence the resonance frequency, as long as the non-linearity introduced by the tie-rods can offset significantly the assessment of the non-linearity of the fiber under testing.
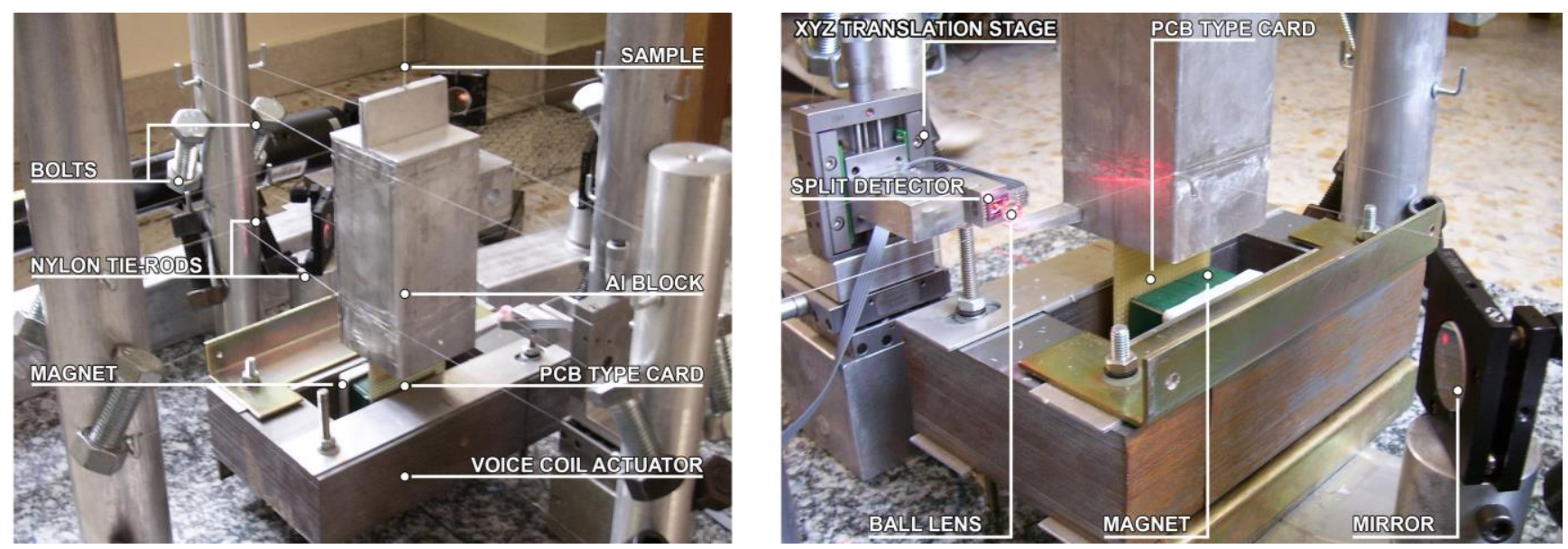

Fig. 2 Significant components in the prototype machine

It is worth pointing out that, when the mass is oscillating, the maximum value of the dynamic angle $\theta$ in equation (1) is given by the oscillation amplitude divided by the length of the tie-rods. This may impose a minimum length for the tierods and/or impose limits on the excitation level when the constraints on the harness disturbance are significant, which is the case for some fibers. In order to guarantee that unloading does not occur, the oscillation amplitude itself must be fixed at a fraction (e.g. 20\%) of the static pretension imposed by the weight of the suspended mass. In turn, this could be up to approximately $20 \%$ of the fiber strength, corresponding to the maximum admissible strain, of which the amplitude is limited within a few percent. The amplitude is typically on the order of $10^{-3}$ for high strength fibers, which suggests rms strains below $10^{-4}$ for the oscillation term. Depending on the length of the fiber sample under testing, such strain variation entails for the suspended mass an rms amplitude with values ranging from below $10 \mu \mathrm{m}$ to above 100 $\mu \mathrm{m}$ for samples of a few centimeters and a couple of meters, respectively. This, in turn, calls for high sensitivity of the vibration detector, which was then designed to yield a sensitivity that was well below a micrometer. Because of this, and the requirement for low disturbance, which suggested the use of a non-contact detector, an optical position sensor was adopted. Interferometry was ruled out because of the limited analog range that it grants and the quiet mechanical 
environment that it necessitates. A focused light approach was used, as illustrated in the following, which yields a sensitivity below $10 \mathrm{~nm}$ in the sine-wave mode without narrow-banding.

In Fig. 3, the methods adopted for non-contact excitation and detection are shown schematically. The vertical forcing tension term is introduced by a voice coil (also observable in Fig. 2) which consists of a magnetic circuit realized via a high saturation transformer type Silicon steel sheet and a 2"x2"x0.5" NdFeB magnet, and a single horizontal wire glued to the bottom of a fiberglass card attached vertically below the mass and inserted into the $8 \mathrm{~mm}$ air gap of the magnetic circuit to position the wire in the center of the magnet. The mass position detection is realized with a ball lens glued to it, which focuses a laser beam onto a split detector. They too are important for the minimization of disturbances that both functions may introduce otherwise.

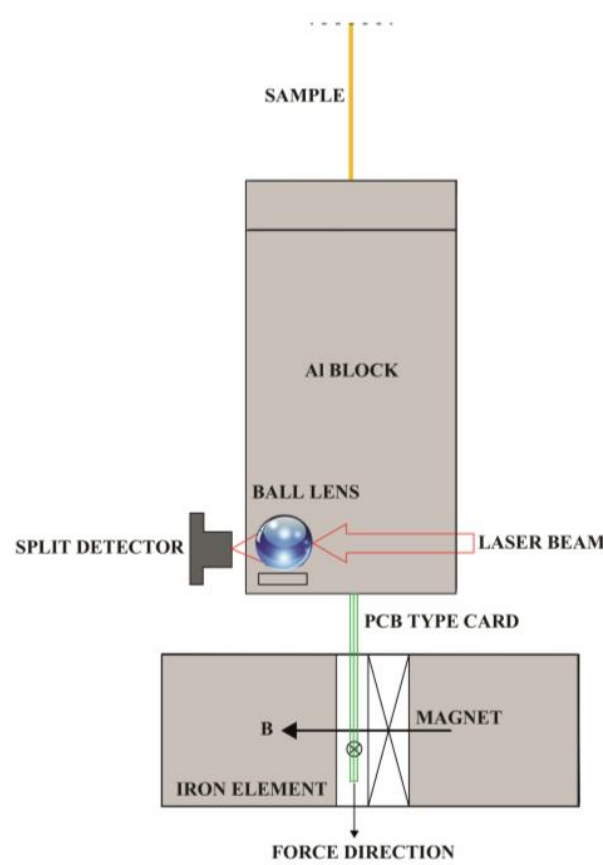

Fig. 3 Non-contact excitation and detection

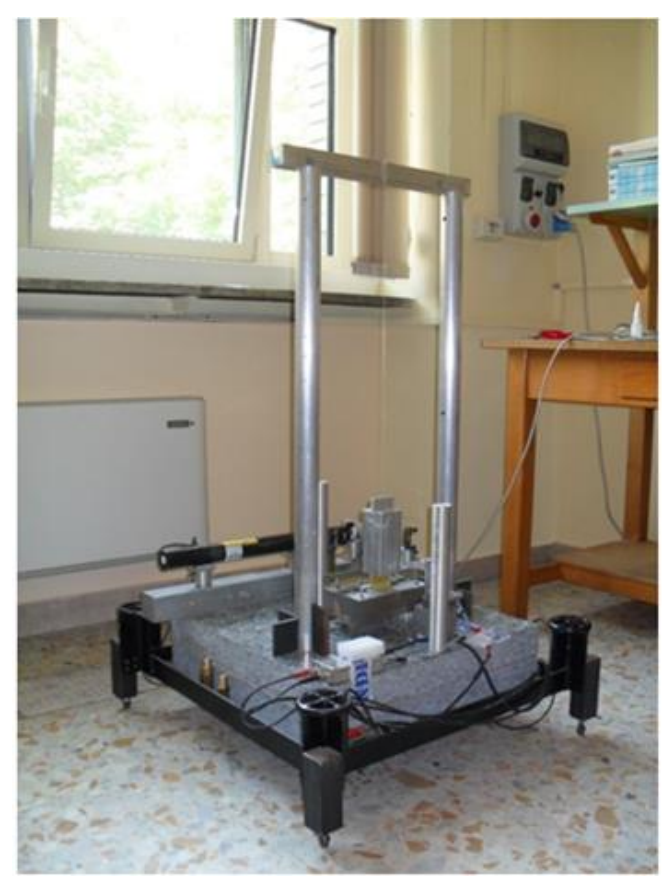

Fig. 4 Prototype testing machine

The expected resonance frequencies are quite low for high strength fibers because of their stiffness. In fact, the resonance angular frequency $\omega_{0}$ can be put in the form

$\omega_{0}=\sqrt{\frac{g}{L \varepsilon_{M}}}=\frac{\omega_{p}}{\sqrt{\varepsilon_{M}}}$,

where $\omega_{p}$ is the free pendulum frequency and $\varepsilon_{M}$ is the fiber strain under the static pre-tension induced by the weight of the suspended mass. Given the mentioned expected values for $L \varepsilon_{M}$, ranging from $50 \mu \mathrm{m}$ to $1 \mathrm{~mm}$ for samples between a few centimeters and a couple of meters, the expected resonance frequency may range between $10 \mathrm{~Hz}$ and $100 \mathrm{~Hz}$, which is actually what was measured for samples of various lengths. 
Different experiments were made with fiber lengths between $40 \mathrm{~mm}$ and $2 \mathrm{~m}$ before the prototype machine was designed and built with its final dimensions.

\section{Prototype description and discussion}

The prototype testing machine is shown in Fig. 4, and consists of a portal with an upper beam from the center of which the fiber specimen is hanging, holding the mass attached at the bottom. The mass is constrained to a vertical degree of freedom by the already mentioned harness of fiber tie-rods. The whole prototype is assembled by simply sticking parts with drops of a cyano-acrilic super-glue, which makes it easy to change configurations and allows for both quick positioning and the necessary assembly stiffness.

The excitation and detection sub-systems are suitably deployed around it. The upper beam of the portal is a half meter long piece of 40x40 mm square section aluminum stock glued and resting on two $0.95 \mathrm{~m}$ tall columns made of $50 \mathrm{~mm}$ diameter, $5 \mathrm{~mm}$ thick aluminum tubing. Both are glued onto a massive $0.6 \times 0.6 \times 0.145 \mathrm{~m}$ granite basement, together with the magnetic circuit of the voice-coil, the optical circuit, and two cylindrical aluminum poles which hold the constraining fiber harness. The tie-rod harness itself is realized with a $0.2 \mathrm{~mm}$ nylon fishing line, as detailed below.

The hanging mass of the resonator system is a $1.375 \mathrm{~kg} \mathrm{Al}$ block, which was not the best choice because of the relatively high acoustic power dissipation during vibrations resulting from its low density, but was handy in the laboratory with a square section shape suitable for harnessing, and sufficient for a prototype. Similar considerations held for the aluminum stock used for the portal. The mass is designed to make the biasing DC stretch of the sample (pretension) a suitable fraction of its tensile strength, so that resonant measurements may be carried out without fully unloading it up to an excitation level, which is useful for non-linearity evaluation.

Sensing of the vertical displacement was realized by means of an optical system, made of a ball lens and a split detector, which had a sensitivity of about $50 \mathrm{mV} / \mu \mathrm{m}$ after amplification of the differential signal. The light source was a $10 \mathrm{~mW}$ HeNe laser that was suitably attenuated to avoid detector damage where the laser power was highly focused by the very fast ball lens. This limited the sensitivity that could be reached. Some care was needed during alignment to obtain the aforementioned sensitivity, which required the use of a XYZ translation stage for positioning the detector in the focus of the ball lens. The lens was attached to the hanging mass and moved with it, displacing, as a consequence, the point at which the laser focuses. 
The observed electronic noise was below $1 \mathrm{mV}(20 \mathrm{~nm})$ without averaging, which is well below the environmental mechanical/seismic noise that instead varied significantly throughout the day on the non-damped table, in all cases being the most important noise source. Averaging helped to reduce its effects, and was adopted in adequate amounts when taking measurements.

The voice coil actuator (60 N/A) consists of a 50x50x12.5 mm NdFeB permanent magnet driving a homemade magnetic circuit realized with an L-shaped laminated transformer type silicon loaded iron and a fiberglass PCB type card fixed vertically below the hanging mass, which held a single $0.5 \mathrm{~mm}$ Copper wire glued horizontally to its lower rim. The sample length was tailored in such a way as to position the conductor in the middle of the $6 \mathrm{~mm}$ gap of the magnet.

The apparatus can be used for both free-decay and forced tests. In the free-decay mode, the ring-down of vertical oscillations is observed by the detector and digitally recorded after A/D conversion. In the forced mode, sine wave excitation is provided through the voice coil by a function generator. The amplitude and phase of the induced vertical oscillation sine wave are measured with an oscilloscope or a vector voltmeter. The sine wave source (AGILENT 3320A, $50 \Omega$ output) reaches, at $1 \mu \mathrm{Hz}, 1 \mu \mathrm{Hz}$ resolution.

\section{The containment web and its effects}

The containment structure is conceived to constrain the oscillating mass in the vertical axis. Its design aimed at maximizing containment and minimizing undesired effects, such as errors in the measured mechanical characteristics of the fiber material under testing. Therefore, an analysis is presented here to guide the design and choice of the material to be used for the tie-rods, and to determine how well the web must be realized.

In the analysis, based on the schematic in Fig. 1, a known equal pre-tension is assumed to be applied to all tie-rods, as produced by the weight of $0.13 \mathrm{~kg}$ bolts, providing a tension of $1.3 \mathrm{~N}$. The generated pre-strain $\mathcal{E}_{\mathrm{t} 0}$ of the tie-rods then depends on their stiffness $k_{t}$, regulated by their length and diameter. To this, an oscillating term $\varepsilon_{\mathrm{r}-\mathrm{rms}}$ is added during operation, because of length changes imposed by geometry, which occurs despite the applied tension, as the mechanism that keeps the tension constant is not fast enough to track oscillations.

For the determination of the sample stiffness, from which the Young modulus $E_{f}$ of the fiber material can be derived, the relevant quantity is the angular frequency $\varpi_{z}$ for vertical oscillations, which is given by

$\omega_{z}=\sqrt{\frac{k_{f}+k_{t z}}{M}}$ 
Its fractional variation induced by the tie-rods harness can be written as

$\frac{\Delta \omega_{z}}{\omega_{z}}=\frac{n}{2} \frac{k_{t}}{k_{f}} \varepsilon_{t 0}$

when taking into account equation (1) and considering that the tie-rods contribution to the vertical stiffness is mainly due to their pre-tension. For accuracy better than $0.1 \%$ of the stiffness, it can be seen from equation (4) that the elastic modulus of the tie-rods should be 10 times smaller than that of the sample for a pre-strain of $3 \times 10^{-4}$. It is assumed here that the tie-rods have roughly the same diameter and one fourth the length of the fiber bundle. For this reason Nylon was used for the tie-rods, as its Young modulus is about $6 \mathrm{GPa}$, compared to an elastic modulus of $80 \mathrm{GPa}$ that is expected for a para-aramid. The length and the diameter of the tie-rods were chosen to be $0.17 \mathrm{~m}$ and $0.18 \mathrm{~mm}$, respectively. It is worth pointing out that, according to equation (4), it is much easier to make the harness irrelevant in the measurement of vertical stiffness when the sample is multifilament than when it is a single fiber. In fact, its stiffness is proportional to the fiber count and this imposes the use of single fibers as tie-rods.

The influence of temperature variations can be easily shown to be minimal, as the thermal expansion coefficient of Nylon is $7.2 \cdot 10^{-5} / \mathrm{K}$ and therefore even an excursion of $14 \mathrm{~K}$ would only produce a variation of $0.1 \%$ on the static strain $\varepsilon_{t}$. According to equation (4) the Nylon harness increases the resonance frequency by $0.2 \%$, which was deemed acceptable, and temperature variations are therefore expected to affect the sixth decimal, which is insignificant.

Attention must be paid to the non-linearity introduced through equation (1) by the oscillating part of the angle $\theta$, which must be added to the static value. In fact, such term introduces a third order coefficient to the system stiffness, which does not belong to the fibers under testing, and represents, therefore, an error contribution when evaluating the nonlinearity of the sample. All tested fibers showed a negative third order coefficient (softening non-linearity), while this dynamic contribution was positive (hardening non-linearity). This supports our confidence in the capability of measuring the non-linearity of fiber material itself.

\section{$Q$ limitations}

The quality factor $Q$ is used to characterize the resonator and its losses, as is customary in forced oscillation measurements. The quality factor $Q=v / \Delta v$ is experimentally obtained as the ratio between the resonance frequency $v$ and full $3 \mathrm{~dB}$ width $\Delta v$ of the amplitude response to excitation, often measured as the frequency difference between the two $\pm 45^{\circ}$ phase shift points on either side of the resonance. The quality factor is inversely proportional to the damping ratio $\zeta$, being $Q=1 / 2 \zeta$, and can be written as 
$Q=\omega_{n} \frac{W}{P_{d}}=2 \pi \frac{W}{W_{d}}$

where $P_{d}$ is the average dissipated power, $W$ is the energy stored in the resonator and $W_{d}$ is the energy lost in one cycle.

Various sources of mechanical energy dissipation contribute to limit the quality factor $Q$ of the spring-mass system. They must be all analyzed in order to assess how well the measured quality factor represents the intrinsic $Q$ of the fiber specimen. The contributions considered here are the energy dissipation in the tie-rods harness, the acoustic radiation, and the dissipation in the equipment producing the forcing term in the forced oscillation technique. The measured $Q$ can, therefore, be seen as the combination of $Q$ limitations, each attributed to one of these mechanisms that would appear as the measured value if the power loss they represent were the only contribution to system dissipation. Such limitations are $Q_{t}, Q_{a}$, and $Q_{e}$, respectively, and their combination is described by the formula

$\frac{1}{Q}=\frac{1}{Q_{f}}+\frac{1}{Q_{t}}+\frac{1}{Q_{a}}+\frac{1}{Q_{e}}$

For the tie-rods' contribution, a comparison with dissipation in the fiber specimens can be done, as both are generated by the same mechanism. In fact, when the spring-mass system oscillates, the strain varies periodically in both tie-rods and fibers under test. In doing so, the energy stored in the oscillator is converted back and forth between kinetic energy stored in the mass and elastic energy stored in the fibers. As a result, part of the converted energy is lost to heat in the process. The lost power is proportional to the power converted by the damping ratio $\zeta$, as well as the energy $W_{d}$ dissipated in a full cycle. Since the elastic energy stored at any time in a stretched string is given by

$W=\frac{1}{2} E A l \varepsilon^{2}$,

the ratio between one-cycle dissipation in the 16 tie-rods $\left(W_{d t}\right)$ and in the fibers under test $\left(W_{d f}\right)$ is

$\frac{W_{d t}}{W_{d f}}=16 \frac{\zeta_{t}}{\zeta_{f}} \frac{E_{t} A_{t} a}{E_{f} A_{f} L} \frac{\varepsilon_{t r m s}^{2}}{\varepsilon_{f r m s}^{2}}$

Introducing the actual values of the setup, namely $E_{t} / E_{f} \approx 0.1, A_{t} / A_{f} \approx 1, a / L \approx 0.25$, and $\zeta_{t} \approx 0.02$ (as separately measured with the same machine), it can be seen from equation (8) that, for an error due to dissipation in the harness smaller than $0.5 \%$ in the measurement of $Q_{f}$ for the extreme case of $Q_{f} \approx 10^{3}$, the dynamic rms strain ratio between tierods and fiber bundle must be smaller than 0.014. This specification is rather easy to satisfy if the tie-rods are perfectly horizontal, because in that case

$\varepsilon_{\text {trms }}=\frac{1}{2} \varepsilon_{\text {frms }}^{2}\left(\frac{L}{a}\right)^{2}$ 
which imposes an easy $\varepsilon_{f r m s}<1.4 \cdot 10^{-3}$ to achieve the desired limit in the error of $Q$. It is harder to meet such a limit if the tie-rods are not perfectly horizontal, but rather inclined by $\theta_{0}$, because in that case:

$\frac{\varepsilon_{\text {trms }}}{\varepsilon_{\text {frms }}}=\theta_{0} \frac{L}{a}$

and, therefore, it must be $\theta_{0}<3.5 \cdot 10^{-3}$, which means that the maximum acceptable vertical misalignment is $0.5 \mathrm{~mm}$. As a conclusion, care is needed to trim the containment web to a configuration in which the dissipation in the tie-rods is not excessively affecting the measurement of $Q_{f}$.

The next contribution to power loss during oscillation is the acoustic radiation from the upper and lower faces of the vibrating mass, as a sound wave propagates away from them. Since the oscillation amplitudes are very small, and so are the velocities, the power of such acoustic radiation can be calculated by the equation

$P_{d, a}=2 \varrho_{0} c S u_{r m s}^{2} \sigma_{r a d}$

in which $\varrho_{0}$ is the air density, $c$ the speed of sound, $S$ the front surface of the vibrating body, $u_{r m s}$ the rms velocity of the radiating surfaces, and $\sigma_{\text {rad }}$ their radiation efficiency. The radiation efficiency depends on the frequency and radiator shape and is taken to be as small as 0.01 for the case at hand, mainly because of the low $20 \mathrm{~Hz}$ frequency [18, $19,20]$.

In this regime, the radiated acoustic power is proportional to the square of rms displacement, exactly the same as the stored energy, which means that the system $Q$ limitation introduced by this mechanism is independent of the oscillation amplitude. Such limit is on the order of $10^{4}$ in the experimental apparatus, which implies an error of $-10 \%$ if $Q_{f}$ is on the order of 1000 . An easy improvement would be introduced by reducing $S$, which can be obtained with a slimmer and denser mass. For example, a somewhat longer and lighter tungsten mass would achieve a reduction by a factor of 10 the cross section $S$, and guarantee an error below $1 \%$ on $Q_{f}$.

With regards to power dissipation in the excitation circuit, the physical mechanism is Joule heating. In fact, the vibration induced e.m.f. dissipates power on the output resistance of the electrical circuit which drives a current into the actuator voice coil, namely the negligible conductor resistance and the relevant $50 \Omega$ output resistance $R_{0}$ of the signal generator. Such dissipated electrical power can be written as

$P_{d e}=\frac{B^{2} \ell^{2} u_{r m s}^{2}}{R_{0}}$ 
where $B$ is the magnetic induction in the magnet gap and $l$ is the length of the exposed wire. In order to evaluate how much it affects the measured $Q$ it must be compared with the input power $P_{i n}=P_{d}$ required to maintain oscillation. The input power is given by:

$P_{\text {in }}=B \ell I_{r m s} u_{r m s}$

where $I_{r m s}$ is the rms current driven into the voice coil, and since

$u_{r m s}=\omega_{n} Q \frac{B \ell I_{r m s}}{k_{f}}$

by considering that $P_{i n} / P_{d e}=Q_{e} / Q$ it can be easily seen that

$Q_{e}=\frac{R_{0} \sqrt{k M}}{B^{2} \ell^{2}}$

Here the term $\sqrt{k M}$ is the characteristic mechanical impedance of the resonator. A quick calculation of this $Q$ limitation yields $Q_{e} \approx 4 \cdot 10^{5}$ for the prototype apparatus, which means that the error introduced by this mechanism in the evaluation of $Q_{f}$ is about $-0.2 \%$ in the case of a fiber with $Q_{f}=1000$. Clearly, a dramatic improvement could be readily introduced by adopting a current source with high output impedance to drive the current into the voice coil, instead of the signal generator at hand. An easy improvement can be made by interfacing the signal generator to the voice coil via a suitable driver stage, which could be a series resistor.

\section{Experimental results}

Measurements of $E, Q$, and non-linearity were taken with the prototype machine on three different kinds of high resistance fibers. Para-aramid fibers, which were measured by laser diffraction to have a diameter of $12 \mu \mathrm{m}$, were a bundle of approximately 2000 unwoven filaments taken from a woven chord. The fiber count was evaluated with the scale and ruler approach from published density values and the measured diameter, and may be argued to be accurate to better than $5 \%$. Silicon carbide (SiC) fiber were $14 \mu \mathrm{m}$ Hi-Nicalon® by COI Ceramics in a tow of 500 fibers, and Carbon fibers were a bundle of about 6000 PAN precursor $7.2 \mu \mathrm{m}$ PX35 fibers by Zoltek ${ }^{\mathrm{TM}}$, taken from a tow of 50000 . The 6000 count evaluation was obtained by comparing bundle sizes with the complete tow through the measured length of sowing threads tight circling either ones ten times, and was judged to be accurate to better than $5 \%$ too. Both original tows were thoroughly specified in their respective data sheets with regards to fiber number and diameter, density and Young modulus, but not for damping. 
In Fig. 5 the results of resonance profiles taken at different excitation levels (lighter blue colors for higher excitation levels) for the three types of fiber are reported as rms strain vs. frequency. The static stress was 60, 176, and 340 MPa respectively, amounting to about $1.6 \%, 6.3 \%$ and $8.2 \%$ of the tensile strength in the three cases. A greater static load easily becomes a problem for the para-aramid fiber because of its high level of creep. For all, the dynamic peak strain was kept below $15 \%$ of the static strain at all times, to avoid unloading and ensure linear operation.

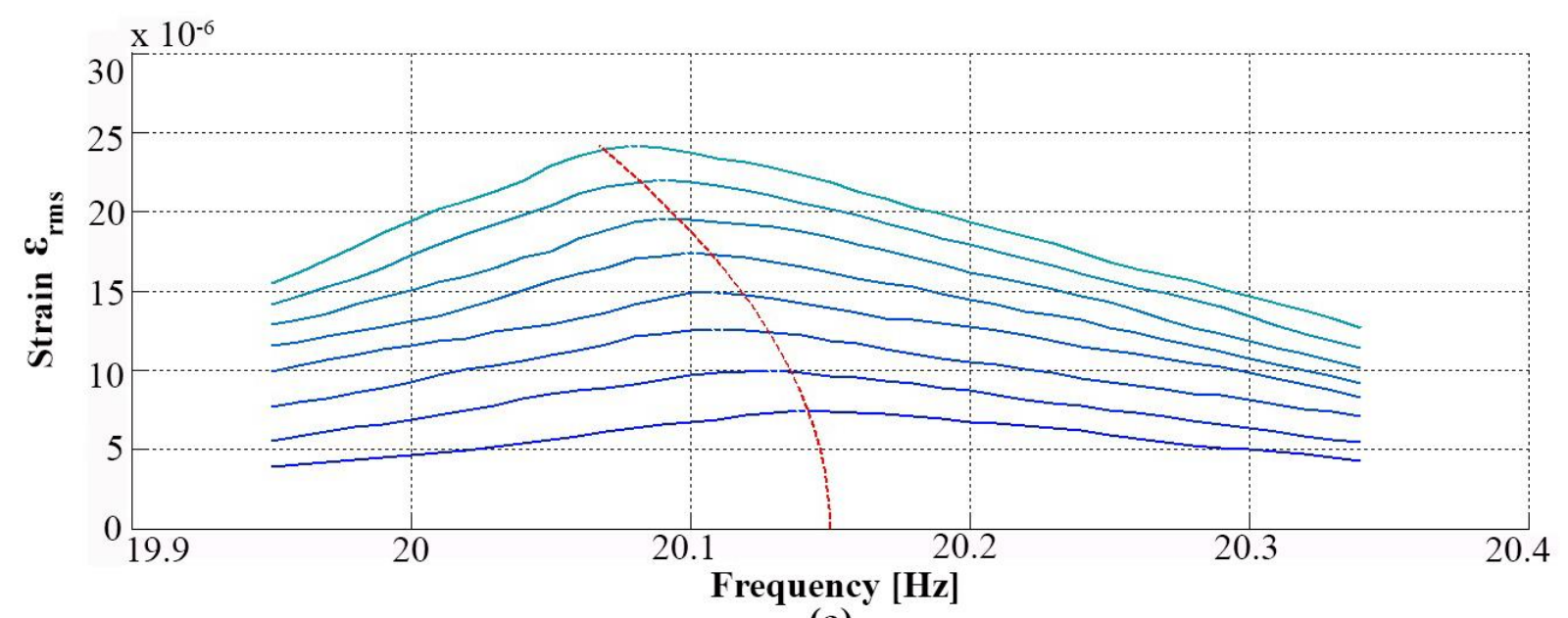

(a)

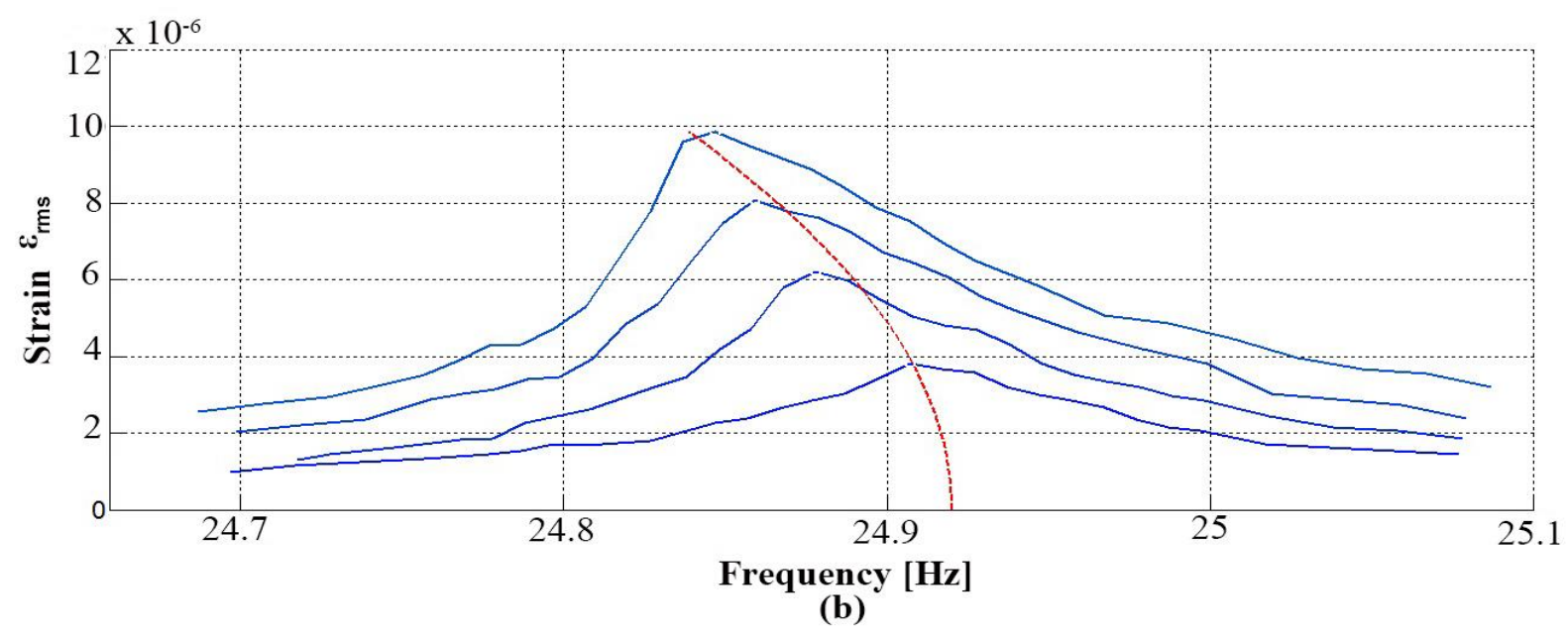




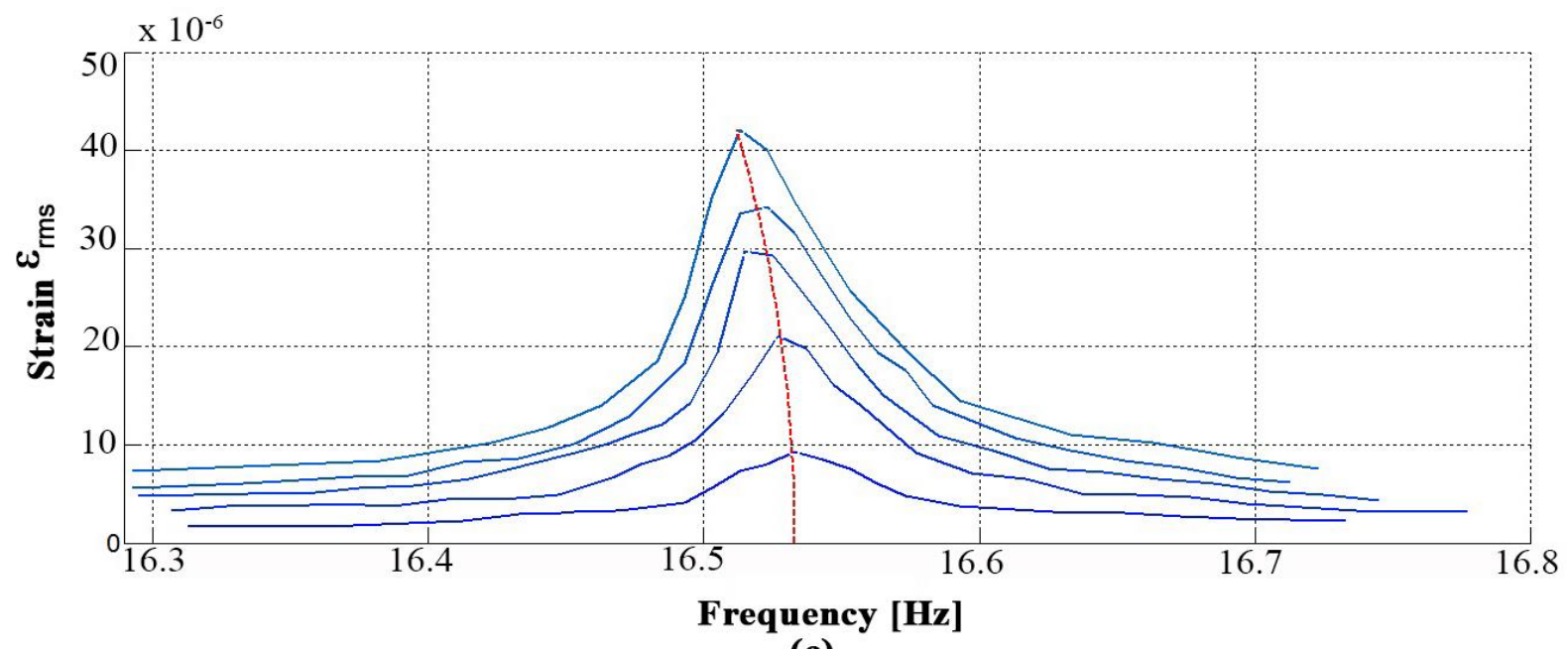

(c)

Fig. 5 Resonance measurement results for the three high resistance fibers: a) para-aramid, $12 \mu \mathrm{m}$ diameter; b) $14 \mu \mathrm{m}$ diameter SiC Hi-Nicalon ${ }^{\circledR}$ by COI Ceramics; c) $7.2 \mu \mathrm{m}$ PX35 carbon fibers by Zoltek ${ }^{\mathrm{TM}}$. Static loading was 60, 176, and 340 MPa, respectively

The analysis of the three families of resonance profiles allows for the extraction of Young modulus, $E$, quality factor, $Q$, and the non-linear coefficient $\chi$ of all three fibers $[21,22,23,24]$. The first quantity is the most uncertain in the prototype apparatus, due to the uncertainty in actual number and diameter of the fibers and because of the difficulty to accurately calibrate the vertical displacement sensor. Nevertheless, all three quantities were in line with data available in literature [25] or from the manufacturer, namely 80, 270, and $240 \mathrm{GPa}$ respectively for para-aramid, $\mathrm{SiC}$, and carbon fibers.

No such comparison could be performed for the $Q$ values and the non-linearity of the three materials, as no data were found in the open literature. The quality factors were measured in a number of different ways, besides the inverse of the $3 \mathrm{~dB}$ relative frequency width of the resonance. Approaches using the phase slope in frequency of the stress to strain transfer function, the 1/e quenching time after cutting the excitation, and the enhancement factor of the strain in resonance were also applied, all yielding pretty much the same values for $Q$. However, the first is certainly the most accurate. Values of 80 for para-aramid, 470 for $\mathrm{SiC}$, and 800 for carbon fibers were asymptotically obtained at low excitation levels, where the asymmetry of the resonance profiles due to non-linearity is less pronounced.

In Fig. 5, the backbone connecting all resonance maxima at various excitation levels is also shown for the three fibers and non-linear coefficients can be extracted from its shape. The values of $-3,02 \cdot 10^{11} \mathrm{~N} / \mathrm{m}^{3}$ (para-aramid), $-2,21$. $10^{12} \mathrm{~N} / \mathrm{m}^{3}(\mathrm{SiC})$ and $-2,08 \cdot 10^{10} \mathrm{~N} / \mathrm{m}^{3}$ (carbon) were found for the cubic non-linear coefficient $\chi$ of the three materials, respectively. It should be pointed out that, as the excitation increased, the backbone always bent to the left, 
towards lower frequencies, for all three materials, clearly indicating that the associated non-linearity was of the softening kind.

\section{Conclusions}

In this paper the principles and realization of a prototype apparatus for fiber testing, capable of accurately measuring material $Q$ values up to $10^{3}$ were illustrated, together with results obtained from three different types of high strength fibers. Suggestions for the improvement of the apparatus are also given, which could extend its accuracy to $Q$ values of several thousands. This apparatus could be a critical tool in the study of mechanical dissipation mechanisms in materials with particularly low levels of intrinsic dissipation.

\section{Acknowledgements}

One of the authors (Andrea De Marchi) would like to acknowledge support of the US Department of Commerce within the NIST Precision Measurement Grant Program (Award ID \# 70NANB15H348). Elena Pinotti is recipient of a "Progetto Giovani” PhD grant funded by the Italian Ministry of Education and Scientific Research, subject: "Advanced structural materials for applications in the cultural heritage field".

\section{References}

1. El Abdi R, Rujinsky A, Borda C, Severin I, Poulain M (2008) New method for strength improvement of silica optical fibres. Optics and Lasers in Engineering 46: 222-229. Doi:10.1016/j.optlaseng.2007.10.005

2. Dulieu-Barton JM, Quinn S, Ye CC (2009) Optical fibre bragg grating strain sensors. In, Eaton Evans, J., DulieuBarton, J.M. and Burguete, R.L. (eds.) Modern Stress and Strain Analysis: a State of the Art Guide to Measurement Techniques. London, GB, Findlay Media, 2-3.

3. JGCM 200: 2012 - International vocabulary of Metrology - Basic and general concepts and associated terms (VIM) - BIPM 2012

4. Botelho EC, Campos AN, De Barros E, Pardini LC, Rezende MC (2006) Damping behaviour of continuous fibre/ metal composite materials by the free vibration method. Composites: Part B 37: 255-263. Doi:10.1016/j.compositesb.2005.04.003

5. Wei CY, Kukureka SN (2000) Evaluation of damping and elastic properties of composites and composite structures by the resonance technique. Journal of Materials Science 35: 3785-3792. Doi: 10.1023/A:1004817011240 
6. Černý M, Glogar P, Manocha LM (2000) Resonant frequency study of tensile and shear elasticity moduli of carbon fibre reinforced composites (CFRC). Carbon 38: 2139-2149. Doi:10.1016/S0008-6223(00)00071-3

7. Nagasankar P, Balasivananadha PS, Velmurugan R (2014) The effect of the strand diameter on the damping characteristics of fibre reinforced polymer matrix composite: theoretical and experimental study. International Journal of Mechanical Sciences 89: 279-288. Doi:10.1016/j.ijmecsci.2014.09.003

8. Berthelot JM, Sefrani Y (2004) Damping analysis of unidirectional glass and Kevlar fibre composites. Composites Science and Technology 64: 1261-1278. Doi:10.1016/j.compscitech.2003.10.003

9. Valtorta D, Lefèvre J, Mazza E (2005) A new method for measuring damping in flexural vibration of thin fibers. Experimental Mechanics 45:433-439. Doi: 10.1177/0014485105056900

10. Rice B, Quinzi J, Lund L, Ulreich J, Shoup M (2014) Measurement of Young's modulus and damping of fibres at cryogenic temperatures. Cryogenics 63: 43-48. dx.doi.org/10.1088/0022-3727/6/1/308

11. Suarez SA, Gibson RF, Deobald LR (1984) Random and impulse techniques for measurement of damping in composite materials. Experimental Techniques 8: 19-24. Doi: 10.1111/j.1747-1567.1984.tb01832.x

12. Suarez SA, Gibson RF (1984) Computer-aided dynamic testing of composite materials. Proceedings SEM Conference on Experimental Mechanics, Milwaukee, WI, USA, p. 118-123

13. Crane RM, Gillespie JW (1991) Characterization of the vibration damping loss factor of glass and graphite fiber composites. Composite Science Technology 40: 355-375. Doi:10.1016/0266-3538(91)90030-S

14. Di Carlo JA, Williams W (1980) Dynamic modulus and damping of boron, silicon carbide and alumina fibers. Presented at the Fourth Annual Conference on Composites and Advanced Materials, Florida.

15. Więcek T (2014) A new method for the measurement of static and dynamic Young's moduli of long fibres. Composites: Part A 60: 1-7. Doi:10.1016/j.compositesa.2014.01.007

16. Gibson RF, Plunkett RA (1977) A forced vibration technique for measurement of material damping. Experimental Mechanics 11: 297-302. Doi: 10.1007/BF02324959

17. Gibson RF, Yau A, Riegner DA (1982) An improved forced-vibration technique for measurements of material damping. Experimental Techniques 6: 10-14. Doi: 10.1111/j.1747-1567.1982.tb01646.x 
18. Larko JM, Cotoni V (2007) Vibroacustic response of the NASA ACTS spacecraft antenna to launch acoustic excitation. Presented at the $14^{\text {th }}$ International Congress on Sound Vibration (ICSV14) cosponsored by the International Institute of Acoustics and Vibration (IIAV) and the Australian Acoustical Society (AAS), Cairns, Australia.

19. Allard JF, Champoux Y (1992) New empirical equations for sound propagation in rigid frame fibrous materials. The Journal of Acoustical Society of America 6: 3346-3353. dx.doi.org/10.1121/1.402824

20. Dahl MD, Rice EJ, Groesbeck E (1987) Effects of fiber motion on the acoustic behaviour of an anisotropic, flexible fibrous material. Prepared for the $113^{\text {th }}$ Meeting of the Acoustical Society of America, Indianapolis, Indiana. dx.doi.org/10.1121/1.398968

21. Demarie GV, Ceravolo R, De Stefano A (2005) Instantaneous identification of polynomial nonlinearity based on Volterra series representation. Key Engineering Materials 293-294: 703-710. Doi: 10.4028/www.scientific.net/KEM.293-294.703

22. Ceravolo R, Erlicher S, Zanotti Fragonara L (2013) Comparison of restoring force models for the identification of structures with hysteresis and degradation. Journal of Sound and Vibration 332: 6982-6999. Doi:10.1016/j.jsv.2013.08.019

23. Masri SF, Sassi H (1982) Identification and modeling of non linear system. Nuclear Engineering and Design 72 : 235-270. Doi:10.1016/0029-5493(82)90219-9

24. Kalmar-Nagy T, Balachandran B (2011) Forced harmonic vibration of a Duffing oscillator with linear viscous damping. In, Kovacic I and Brennan M. (Eds.) The Duffing Equation: Nonlinear Oscillators and Their Behaviour. John Wiley \& Sons.

25. Hollaway L, 1993. Polymer Composites for Civil and Structural Engineering. Chapman \& Hall, London. 
2016-09-14

\title{
A new testing machine for the dynamic characterization of high strength low damping fiber materials
}

\author{
Ceravolo, Rosario
}

Springer

Rosario Ceravolo, Andrea De Marchi, Elena Pinotti, Cecilia Surace, Luca Zanotti Fragonara. A new testing machine for the dynamic characterization of high strength low damping fiber pÿmaterials. Experimental Mechanics, January 2017, Volume 57, Issue 1, pp. 6574 http://dx.doi.org/10.1007/s11340-016-0208-4

Downloaded from Cranfield Library Services E-Repository 